\title{
Prediction of Energy Effects on Photovoltaic Systems due to Snowfall Events
}

\author{
Rob W. Andrews ${ }^{1}$ and Joshua M. Pearce ${ }^{2}$ \\ ${ }^{1}$ Department of Mechanical and Materials Engineering Queen's University, Kingston, Ontario, Canada \\ ${ }^{2}$ Department of Materials Science and Engineering \& Department of Electrical and Computer Engineering, \\ Michigan Technological University, Houghton, MI, USA
}

\begin{abstract}
The accurate prediction of yields from photovoltaic systems (PV) is critical for their proper operation and financing, and in northern latitudes the effects of snowfall on yield can become significant. This work provides methods for identifying snowfall effects from commonly collected performance data, and recommends a model to allow for prediction of these effects based solely on meteorological time series. The model was validated with data from two large-scale ( $>$ 8MW) operational PV plants. For the low tilt angles most affected by snowfall, this analysis was able to accurately predict both daily and mean values of snow effects. This methodology will enable system operators to utilize performance data to accurately identify and predict snowfall losses, and will assist system designers to optimize for the effects of snowfall on new system designs.
\end{abstract}

Index Terms-PV modelling, Snowfall, Photovoltaics, Regressive modelling, large scale PV

\section{INTRODUCTION}

As the levelized cost of solar electricity decreases [1], the momentum in the photovoltaic (PV) industry continues to grow and the global pattern of system implementation is spread among a variety of latitudes away from the ideal equatorial regions where the solar resource is maximized. Recent work has identified large surface areas at high latitudes appropriate for PV [3], [4]. As such roughly 74\% of PV resources were installed in countries that experience some amount of snowfall [5]. Depending on the orientation of the PV modules and meteorological factors, previous studies have indicated that snow losses on a PV system can be as high as $20 \%$ for a low profile system to $0.3-2.7 \%$ for a highly exposed 28 degree roof mounted system [6]-[11]. A comprehensive recent study has attempted to create a regressive model to predict the losses due to snowfall, however there was a large demonstrated scatter in the dataset, which is a limitation when attempting to create a generalizable model for snowfall losses [12]. The ability to accurately perform these predictions is needed by the industry in order to improve financial projections and obtain favourable project financing.

This paper will first utilize data collected from the recently commissioned Open Source Outdoors Test Field (OSOTF) [13], in order to demonstrate methods for identifying snowfall effects and the development of the proposed model. Data from two large scale $(>8 \mathrm{MW})$ operational PV plants located across southern Ontario will then be utilized to validate this model and to demonstrate its applicability.

\section{BACKGROUND}

Snowfall effects the performance of a PV module with two opposing mechanisms. The first, and most obvious, is the collection of snow on the surface of a module which causes a decrease in the light impacting the face of the module. The second mechanism causes an increase in PV relative performance due to the increased albedo present in the surroundings, which can reach a spectrally weighted value of close to $94 \%$ for fresh snow with crystalline silicon (c-Si) based modules [14].

Previous snow studies have relied on continuous clearing of a set of control modules [12] or on the comparison of actual system output to modelled system output over a given time period [6], which is the methodology adopted by this work. It has, however been shown that many accepted modelling techniques can have a large portion of seasonal and absolute bias due to spectral effects and module power tolerances, in addition to not properly accounting for albedo irradiation. Therefore, this work uses the adaptive modelling algorithm proposed in [15] to reduce these biases and to provide high resolution estimates of ideal system output.

\section{DATA COLlection}

\section{A. Open Source Outdoors Test Field (OSOTF)}

A selected set of 15 modules from the OSOTF were used in the analysis. These modules are installed at angles of $10^{\circ}$, $20^{\circ}, 40^{\circ}$, and $70^{\circ}$ with four modules installed at each angle, with the exception of $20^{\circ}$ set, which has three modules. All modules are $\mathrm{c}-\mathrm{Si}$, however each module at a given angle is from a unique manufacturer or incorporates a specialized surface coating. Short circuit current $\left(I_{s c}\right)$ is measured using a current shunt and module temperatures are measured with type $\mathrm{T}$ thermocouples at 5-minute intervals. In addition, global $\left(G_{t}\right)$ and diffuse $\left(D_{t}\right)$ irradiation are measured with two Kipp \& Zonnen CMP 22 pyranometers and snow depth is measured using an ultrasonic snow depth sensor. However, in order to maintain consistency in the data sources between the OSOTF and solar farm measurements, snowfall data from the nearby Kingston climate weather station [16] was utilized. Data was collected for the winters of 2010/2011 and 2011/2012. 


\section{B. Validation with Multi-MW Solar Farms}

Hourly data was collected from two large commercially operational solar farms, designated SF1 and SF2, which were within a $20 \mathrm{~km}$ radius of each other and a $50 \mathrm{~km}$ radius of the nearest WMO certified weather station. Snowfall and other meteorological data were derived from this weather station, and DC power input to each inverter (single MPPT, $500 \mathrm{~kW}$ DC), Solar irradiation (2X CMP 11 Pyranometer), and module temperature measurements were collected from each site.

\section{Generation of OSOTF snow losses}

For the individual modules at the OSOTF, a modified version of the Sandia PV array performance model was used to predict their output [17], as described in [15]:

$$
\begin{aligned}
\hat{I}_{s c}= & \frac{I_{s c o}}{1000} \phi_{I s c o} \cdot\left(B_{t} \cdot f(A O I) \cdot f(A M)+\ldots\right. \\
& \left.\ldots+D_{t} \cdot f\left(K_{t}\right)+A_{t}\right) \cdot\left[1+\alpha\left(T_{c}-T_{o}\right)\right]
\end{aligned}
$$

Where $\hat{I}_{s c}$ is the predicted short circuit current of the module, $I_{s c o}$ is the manufacturers rated short-circuit current at STC, $B_{t}, D_{t}$, and $A_{t}$ represent the beam, diffuse and albedo components of irradiation, $f(A O I) f(A M)$ and $f\left(K_{t}\right)$ are functions accounting for reflectance losses, air mass and clearness ratio respectively, $T_{c}$ and $T_{o}$ represent the cell and reference temperature respectively, $\alpha$ is the module short circuit temperature coefficient, and $\phi$ is an empirically derived coefficient which accounts for module degradation and soiling. $\phi$ is derived using a least squares optimization routine, which minimizes the errors between $\hat{I}_{s c}$ and $I_{s c}$. Points were chosen for this routine which were at least three days from the presence of snow on the ground, and within three days of a rainfall of at least $2 \mathrm{~mm}$, in order to attempt to reduce the effects of soiling.

Snow losses were defined over the periods where snowfall was recorded as $I_{s l}=\hat{I}_{s c}-I_{s c}$. This value was then integrated over an entire day to determine the daily effect in photocurrent due to snowfall. The daily change in snow depth $\left(\bar{S}_{t}\right)$ was calculated as the difference between the morning and night-time values, giving the accumulation or reduction in snow depth over the day.

\section{Generation of large farm snowfall losses}

Two secondary standard pyranometers were available per testing site to record Plane Of Array (POA) irradiation. However because of the large number of modules on each MPPT string (greater than 2,000), the stochastic effects of cloud cover effected the point measurement from the pyranometers to a greater degree than the averaged output of the strings. Because of this the Root Mean Squared Error (RMSE) of the correlation between pyranometer data and string output was greater than that at the OSOTF where each individual module was measured. In addition, a degree of DC clipping occurred as the strings were intentionally oversized for the inverters. Therefore, when developing a parameteric empirical model for module output, only periods where DC clipping was not present were utilized. Due to the low temporal resolution of the dataset, spectral correction was not applied. The model used to predict string output is thus an adaptation of equation 1 for power output:

$$
\hat{P}_{m p}=\frac{P_{S T C}}{1000} \phi_{P_{s t c}} \cdot\left(G_{t} \cdot f(A O I)\right) \cdot\left[1+\alpha\left(T_{c}-T_{o}\right)[2)\right.
$$

Where $\hat{P}_{m p}$ is the power at the maximum power point, $P_{S T C}$ is the STC rating of the modules, and $\phi_{P_{s t c}}$ is an under performance modifier that was determined through a least squares method similar to that used for $I_{s c}$. However, because of the higher error in the resultant modelled output due to the increased effects of stochastic shading, there was a larger issue of noise effecting the proper determination of snowfall losses. To remedy this, low level noise was eliminated by selecting only those points where $P_{s l}=\hat{P}_{m p}-P_{m p}$ was greater than its standard deviation. The validity of this assumption can be seen in the results.

\section{Statistical Analysis}

Using the OSOTF a first attempt was made to directly correlate the module energy gain or loss in a day to the change in the level of snowfall on that day, however as shown in Figure 11, there was no apparent correlation between daily snowfall and amount of photocurrent loss at any tilt angle.

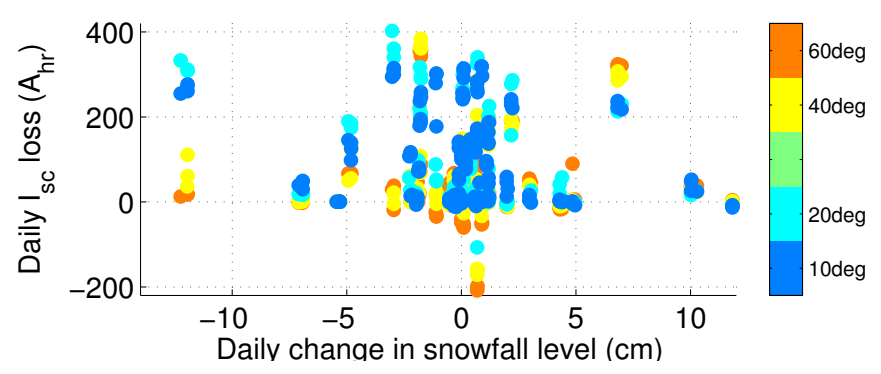

Fig 1: Correlation of daily snowfall to snow losses.

In order to account for this, and based on practical observations, it was hypothesized that there is a level of persistence in the snow effect data, where the effect on a given day would be somehow related to the losses of a previous day, due to the build-up of snowfall on the module. In order to investigate this, time series analysis techniques as outlined in Brockwell and Davis [18], were used to calculate the partial autocorrelation (PACF) and autocorrelation (ACF) of the snow effect time series. The results are displayed as the surface shown in Figure 2. From the significant values of the ACF and PACF at lag 1 seen in Figure 2, it can be seen that there is some time correlation of the datasets, related to a lag of one day, which decreases as a function of angle. In order to attempt to explain this correlation, PACF of snowfall time series data from the OSOTF rooftop are shown in Figure 3

From Figure 3 it can be seen that the daily snowfall change data shows the characteristics of a stochastic white noise process. Therefore, the correlation seen in the snow 

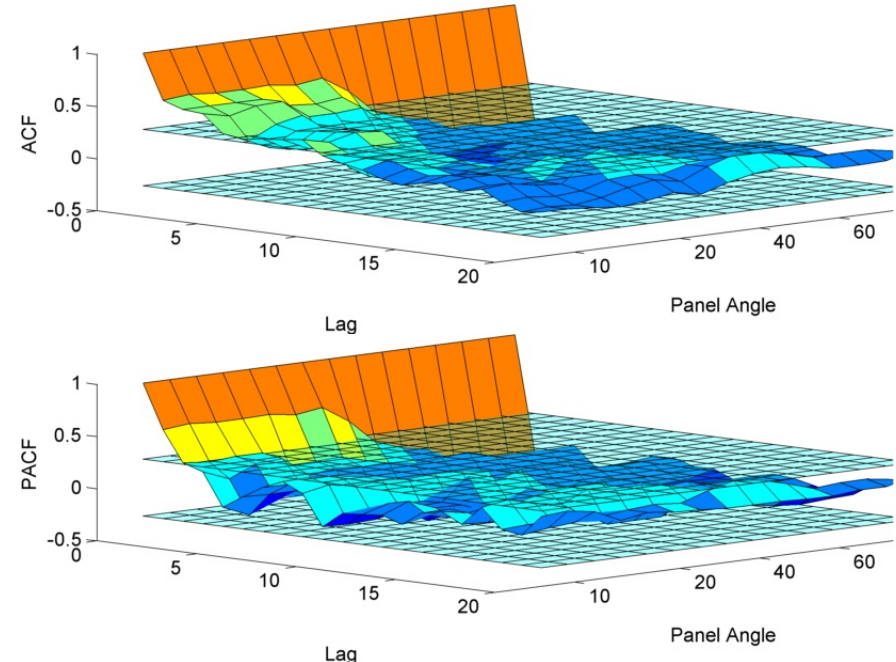

Fig 2: ACF and PACF of snow effect data. The horizontal surfaces represent significance bounds on the correlation in time.

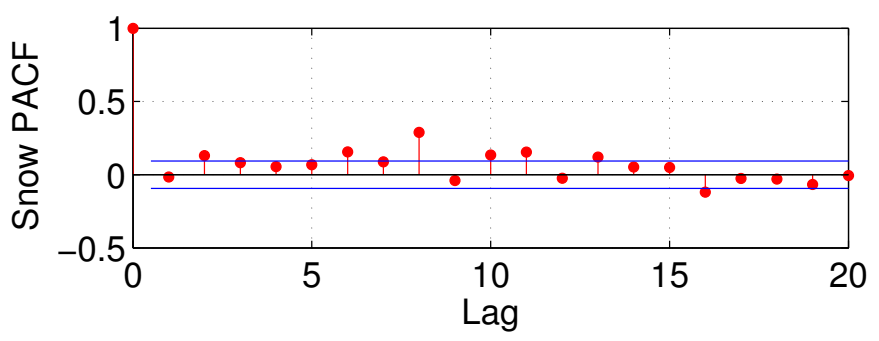

Fig 3: Partial autocorrelation function of the snowfall data for the OSOTF for 2010/2011.

effect data set is likely from another mechanism, which will be investigated below.

\section{Model Development}

Based on the statistical analysis it can be seen that a model including a scaled historical dependence on a white noise process, in this case change in snow depth, should be utilized. From previous investigations, [9], [12], [19] and observations, it was seen that average temperature $\left(\bar{T}_{t}\right)$, relative humidity and wind speed will tend to affect the likelihood of snow to shed in a day. However, upon performing a sensitivity analysis using the RMSE of the model, it was found that relative humidity and wind speed and were not significant. In addition, the magnitude of energy gain or loss from snowfall will be proportional to the mean solar irradiation in a given day. Based on these assumptions the following snow effect model is proposed for short-circuit current:

$$
\hat{I_{s l}}=\psi_{1} * \bar{S}_{t}+\psi_{2} * \bar{G}_{t}+\psi_{3} * \bar{T}_{t}+\psi_{4} * \bar{S}_{(t-1)}
$$

And for Power:

$$
\hat{P_{s l}}=\psi_{1} * \bar{S}_{t}+\psi_{2} * \bar{G}_{t}+\psi_{3} * \bar{T}_{t}+\psi_{4} * \bar{S}_{(t-1)}
$$

Where $\bar{S}_{t}$ is the positive change in depth of snow on the ground. This was filtered to include only days with an increase greater than $1 \mathrm{~cm}$,as this is the smallest resolution measured at Environment Canada weather stations,and the inclusion of $1 \mathrm{~cm}$ snowfalls introduced a large source of noise into the data. In order to improve the generalizability of this model, the array of input snow loss data is normalized by the STC power rating of the device. In the case of the short-circuit current this was approximately 8A and in the case of the farm inverters this was $500 \mathrm{~kW}$. The resultant units for daily loss are in hr of STC illumination equivalent.

The coefficients $\psi \in\left\{\psi_{1}, \ldots, \psi_{4}\right\}$ were evaluated using a least squares data fitting algorithm. An example of a predicted time series is shown in Figure 4 for short-circuit current over a day and in Figure 5 for utility scale power over the winter of 2010/2011.
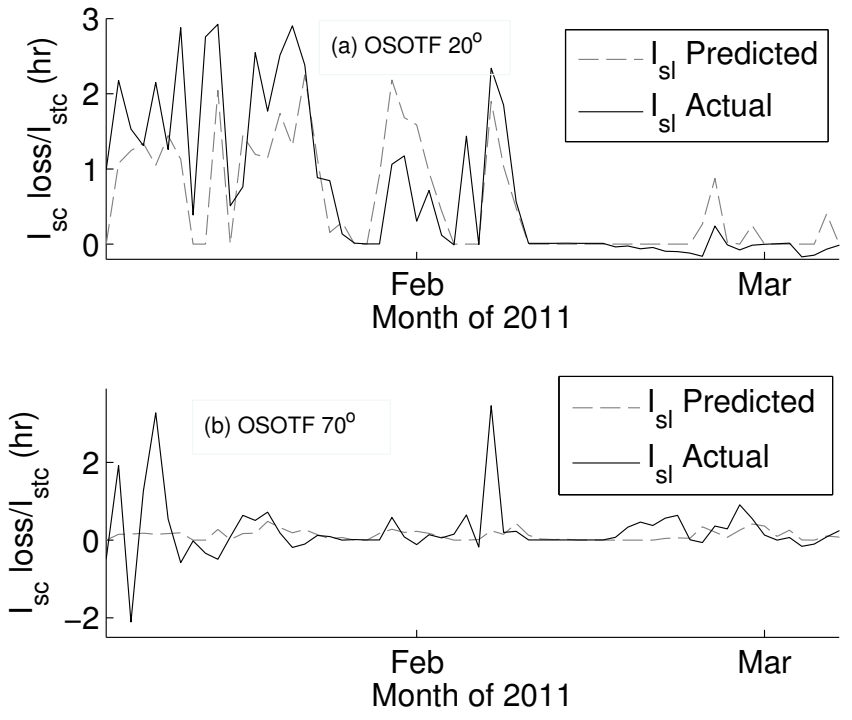

Fig 4: Model fit time series for a $20^{\circ}$ (a) and $70^{\circ}$ (b) tilt angle. Note the effects of albedo reflection on the higher angle modules, represented by negative power losses.

It should be noted that the most major spikes in the predicted snow loss $\left(\hat{P_{s l}}\right)$ are captured in the actual snow loss $\left(P_{s l}\right)$ These two datasets were derived from separate sources, the actual losses from electrical performance of the system and the predicted losses from meteorological data. Thus it can be seen, he previous assumption is valid that the only losses which will count as being caused by snow should be above the standard deviation of modelling errors.

\section{Vi. Model Evaluation}

A comparison of actual and predicted daily snowfall effects across selected modules from the OSOTF is shown in Figure 6 Though there is a good correlation at low angles, it can be seen that at a daily resolution, the prediction of snow effects for high angle modules is very limited. The important attribute for a systems designer, however, is the total snowfall effect in a year, and as such the fractional loss of power over the entire year of production are compared to modelled values in Figure 

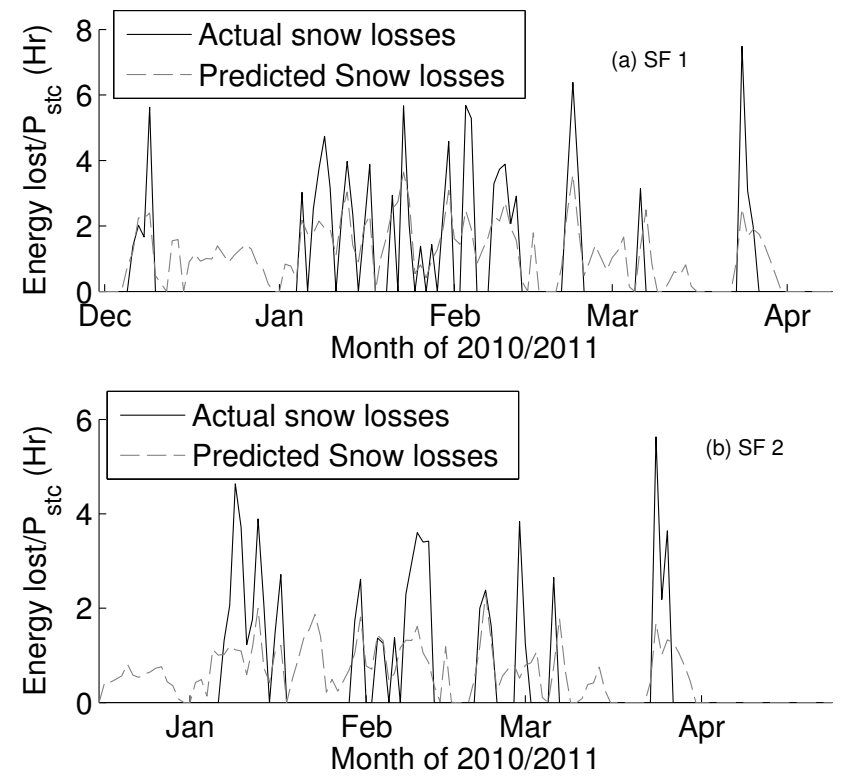

Fig 5: Timeseries of model fits for winter of 2010/2011 to a represenative inverter from a solar farm for SF1 (a) and SF2 (b).

7. It can be seen that the error in predicting the true mean snowfall effect based solely on meteorological measurements is relatively low for low tilt angles, which experience large magnitudes of snowfall effects.

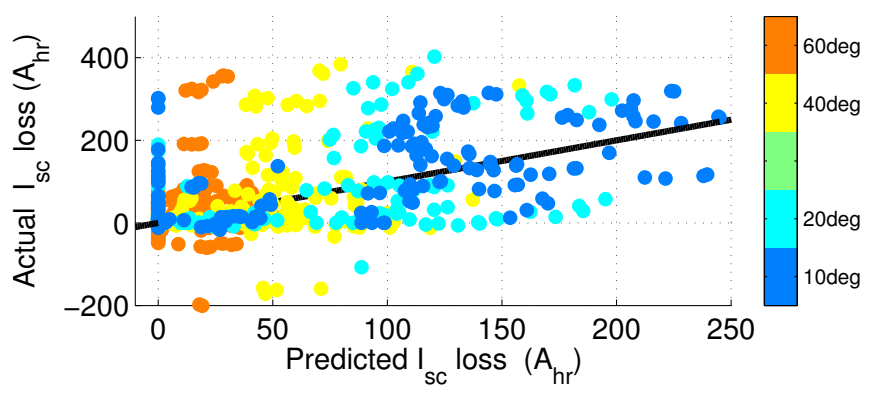

Fig 6: Comparison of modelled and actual module snowfall effect. The line represents a perfect model fit.

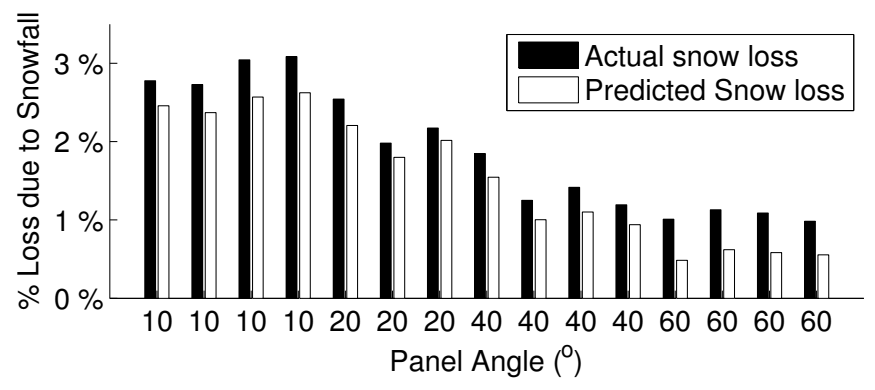

Fig 7: Percent error in predicted mean snowfall effect on photocurrent loss.

In order to evaluate the suitability for generalization and stability of the model, the normalized coefficients $\psi \in\left\{\psi_{1}, \ldots, \psi_{4}\right\}$ are shown in Figure 8 . It can be seen that there is some angular dependence to the coefficients at the OSOTF, and that at SF1, the coefficients are relatively stable across all inverters, which is to be expected as they are experiencing the same meterological phenomenon.
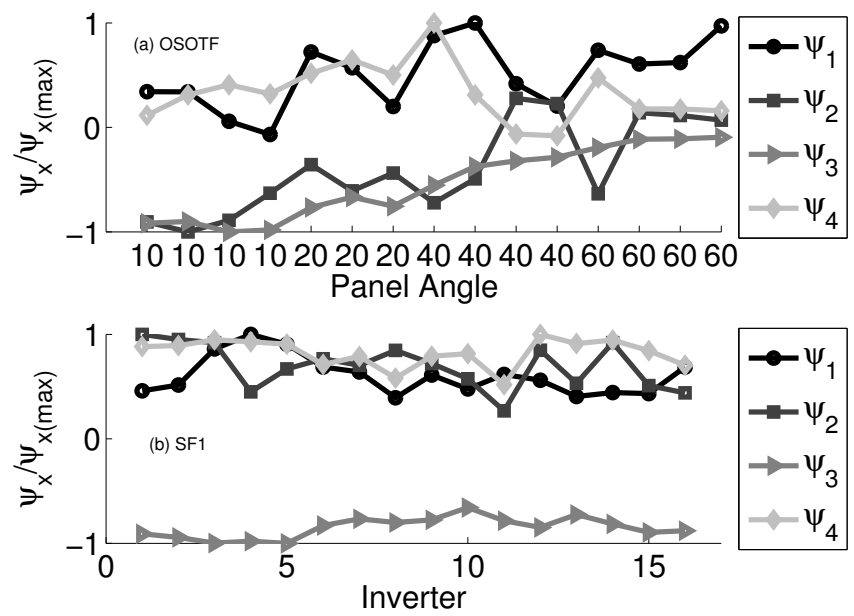

Fig 8: Normalized values of model coefficients. (a) shows some angular dependancy on the model coefficients and (b) shows that across all inverters the model coefficients are relatively stable.

Figure 9 shows a comparison of the derived model coefficients from the three sources used in this report. It can be seen that they are generally stable and in agreement, with the exception of $\psi_{2}$. This parameter relates to the effect of daily insolation on snow losses. One possible reason for this large discrepancy is the previously noted discrepancy in module averaging between the OSOTF and the large farms. Because insolation is more weakly related to module output in the large farms due to the increase of stochastic errors, the value of the parameter may be effected.

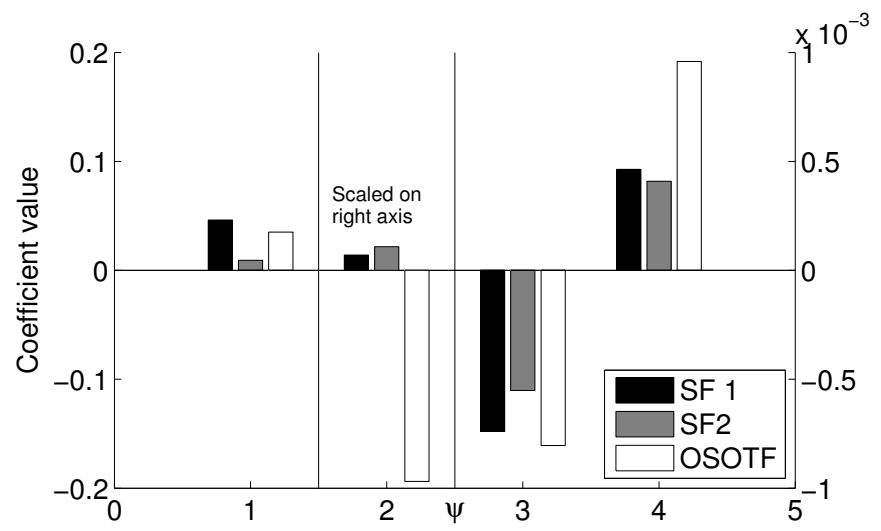

Fig 9: Comparison of model coefficients derived from DH1, DH2 and the OSOTF.

\section{Application to Systems Design}

This methodology was applied to the output of all inverters of SF1 and SF2, and a typical output for a half of a year for one these inverters is seen in Figure 10, which 
shows significant snow losses in the winter and DC clipping losses starting in February and continuing through the summer. On application of this model, the actual and predicted snow losses are shown in Figure 11

It can be seen that the snow losses measured for the large farms are greater in magnitude to those measured at the OSOTF. One mechanism for this is due to the large number of modules located on a single Maximum Power Point Tracking (MPPT) circuit. Because of this, if only a few modules are obstructed by snow, it will reduce the performance of the entire circuit, which could magnify the snowfall losses on a string. Also, because the modules are intentionally oversized for a given inverter the fraction of energy production in the wintertime is proportionally larger than if the clipped power in the summer time was produced. Because of this, snow losses will account for a larger fraction of total produced energy.

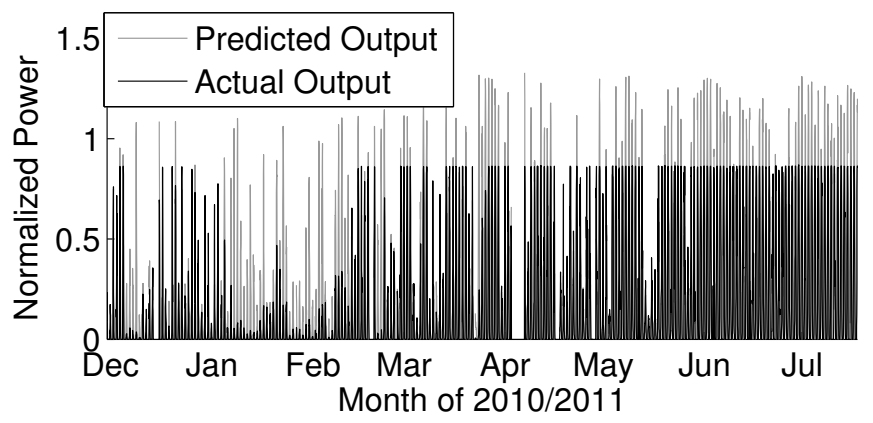

Fig 10: Timeseries showing typical performance of a single inverter in a solar farm.
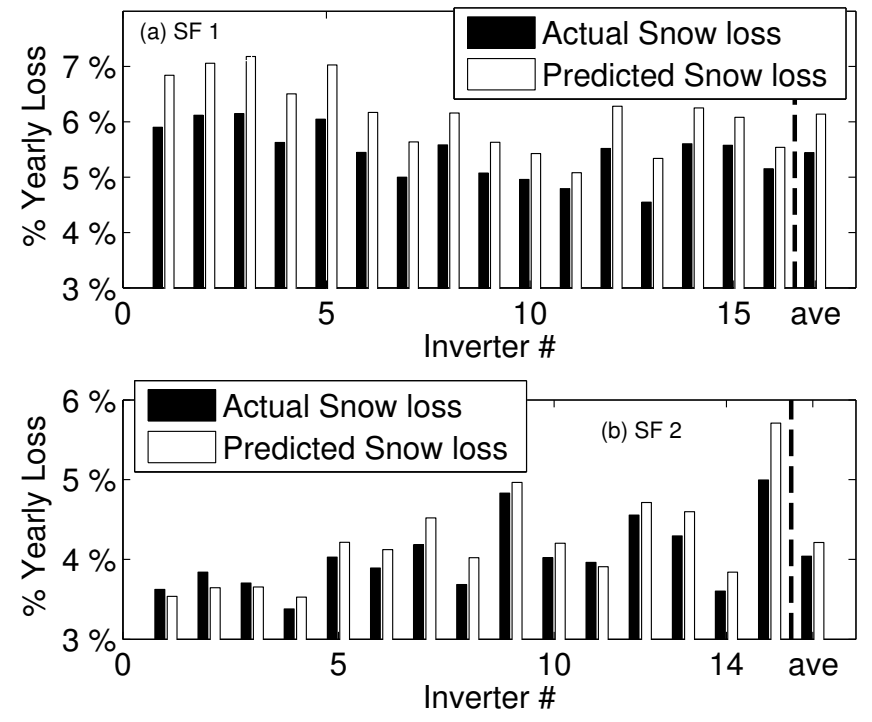

Fig 11: Comparison of actual and modelled snow losses for SF1 (a) and SF2 (b).

To give an estimate of the value of these snow losses, assume that a typical system in Ontario will produce energy at $1200 \mathrm{kWh} / \mathrm{kW}$ [3]. For an $8 \mathrm{MW}$ farm, this amounts to 9.6 million $\mathrm{kWh}$ of yearly production. Sold to the grid at current Ontario Feed in Tariff rates of $\$ 0.347 / \mathrm{kWh}$ this amounts to a yearly revenue of $\$ 3.3$ million. A $4.25 \%$ loss of energy due to snowfall therefore amounts to a yearly loss of $\$ 140,000$ per site.

Of primary importance to a systems designer, however, is the prediction of snow losses for a system that has not yet been created. It would therefore be desirable to generate a set of coefficients from a similar system, and utilize the coefficients to predict the losses which will be seen from the system being designed. In order to test the applicability of this approach, the snow losses for SF 2 were determined using the coefficients derived from SF 1 and the OSOTF, and the results of this are seen in Figure 12

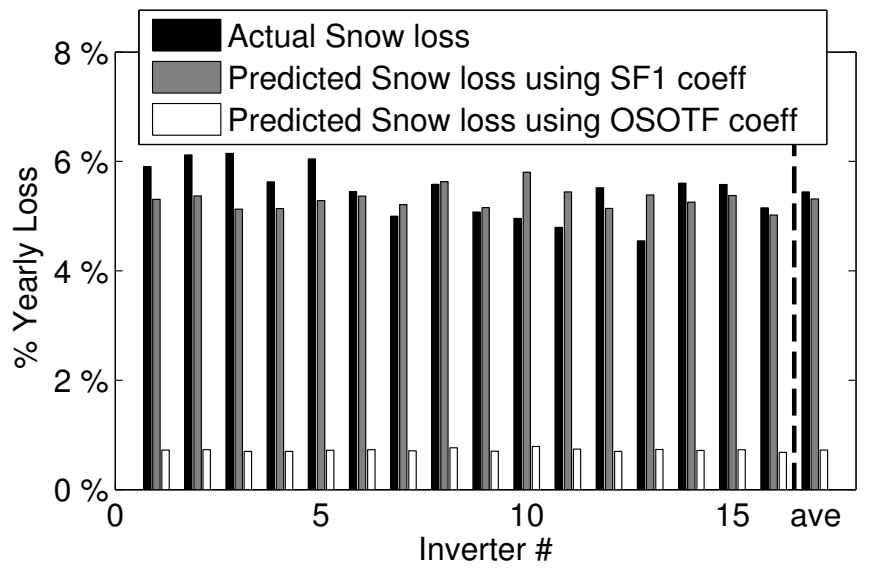

Fig 12: Applicability of snow loss coefficients to prediction of SF2 snow losses.

It can be seen in Figure 12 that coefficients from the similar system provide a good estimate (within $3 \%$ ) of annual snow losses. The quality of this fit is also likely improved by the close proximity of the two sites, and it would be desirable in the future to integrate data from a geographically disparate system of a similar configuration in order to compare performance predictions.

Finally, in order to give context to a site, an evaluation of historical data should be performed. Figure 13 shows the predicted snow losses for sites SF1 and SF2 using historical data from 1997-2009 for both sites. Historical solar irradiation data was not available, and so all losses are referenced to predicted 2011 yields.

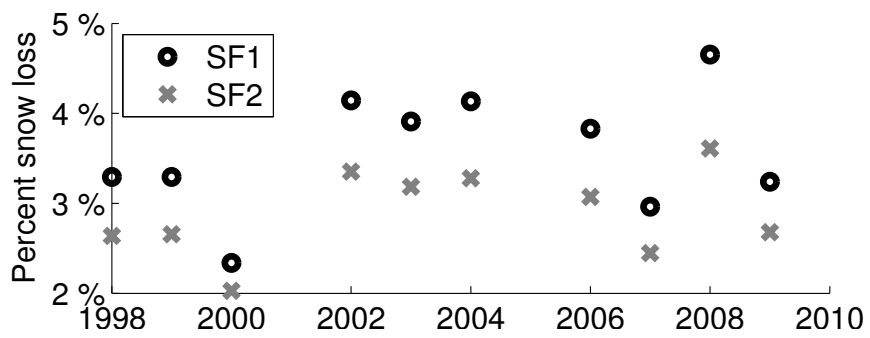

Fig 13: Application of snow loss coefficients to historical datasets, showing annual trends. 


\section{FUTURE WORK AND EXTENSIONS}

It has been shown that snow losses for distributed sites of a similar type can be predicted using this methodology. This is the beginning of the development of this methodology, and the addition of more representative systems will broaden its applicability. The addition of data from flush mounted rooftop systems would require adjustments to the model to incorporate ground effects, and would further broaden the applicability of the approach. In order to perform this analysis only normalized data and a rough geographical location are required, and the results can be beneficial to the entire industry. Therefore it can be seen that the sharing of normalized non-proprietary data within the industry will enable further development and innovation in the field, which is not achievable by a single company, and further investigations into methods to enable open data sharing should be performed.

\section{CONCLUSiOnS}

The analysis presented in this paper outlines a methodology for predicting the losses on a PV module based on readily available meteorological data for the specific site. For low tilt angles most affected by snowfall, this analysis was able to accurately predict both daily and mean values of snow effects. Using the methodology proposed in this paper, it is possible for a systems operator to extract information on system snow losses based solely on a system performance time series, and use this information to create a predictive model for snow losses for the existing and similar PV sites. In addition, this methodology can be used with a large dataset to derive an accurate, generalizable model for snow effects prediction based on actual meteorological time series data, to be used for more accurate modelling of PV systems.

\section{ACKNOWLEDGEMENTS}

The authors would like to acknowledge the work of $\mathrm{H}$. McLaren, J. Fairborn, Q. Richardson, D. Carter and A. Babasola and the support of SEARC at St. Lawrence College, and to our industry partners. In addition we acknowledge support from the NSERC and SSHRC.

\section{REFERENCES}

[1] K. Branker, J. Pathak, and J. M. Pearce, "A review of solar photovoltaic levelized cost of electricity," Renewable \& Sustainable Energy Reviews, vol. 15, pp. 4470-4482, 2011.

[2] D. W. McKenney, S. Pelland, Y. Poissant, R. Morris, M. Hutchinson, P. Papadopol, K. Lawrence, and K. Campbell, "Spatial insolation models for photovoltaic energy in canada," Solar Energy, vol. 82, no. 11, pp. 1049-1061, Nov. 2008. [Online]. Available: http://www.sciencedirect.com/science/article/ B6V50-4SM6R74-2/2/f3f32a0a349845fd72b0e384ba042ab8

[3] H. Nguyen and J. M. Pearce, "Estimating potential photovoltaic yield with r.sun and the open source geographical resources analysis support system," SSRN eLibrary. [Online]. Available: http://papers.ssrn.com/sol3/papers.cfm?abstract_id=2006712

[4] SolarBuzz, "MarketBuzz," Tech. Rep., Mar. 2010.
[5] G. Becker, B. Schiebelsberger, W. Weber, C. Vodermayer, M. Zehner, and G. Kummerle, An Approach To The Impact Of Snow On The Yield Of Grid Connected PV Systems. Bavarian Association for the Promotion of Solar Energy, Munich, 2007.

[6] B. L. Brench, "Snow-covering effects on the power output of solar photovoltaic arrays," DOE DE-AC02-76ET20279, Dec. 1979. [Online]. Available: http://adsabs.harvard.edu/abs/ 1979STIN...8111551B

[7] B. Marion, J. Adelstein, K. Boyle, H. Hayden, B. Hammond, T. Fletcher, B. Canada, D. Narang, A. Kimber, L. Mitchell, G. Rich, and T. Townsend, "Performance parameters for GridConnected PV systems," 31st IEEE Photovoltaics Specialists Conference and Exhibition, Jan. 2005. [Online]. Available: http://ieeexplore.ieee.org/stamp/stamp.jsp?arnumber=01488451

[8] M. M. D. Ross, "Snow and ice accumulation on photovoltaic arrays: An assessment of the TN conseil passive melting technology, report\# EDRL 95-68 (TR), energy diversification research laboratory, CANMET," Natural Resources Canada, Varennes, September, 1995.

[9] H. Yoshio, S. Toshiyuki, and T. Makoto, "Snow fall on the photovoltaic array and snow sliding condition." Journal of Snow Engineering of Japan, vol. 15, no. 4, pp. 15-16, 1999. [Online]. Available: http://sciencelinks.jp/j-east/article/200002/ 000020000299A1018695.php

[10] K. Yoshioka, T. Saitoh, and T. Yamamura, "Performance monitoring of a building-integrated photovoltaic system in an urban area," in Photovoltaic Energy Conversion, 2003. Proceedings of 3rd World Conference on, vol. 3, 2003, pp. 2362-2365 Vol.3.

[11] T. Townsend and L. Powers, "Photovoltaics and snow: An update from two winters of measurements in the sierra," 2011.

[12] J. M. Pearce, A. Babasola, and R. Andrews, "Open solar photovoltaic systems optimization," Proceedings of the 16th Annual National Collegiate Inventors and Innovators Alliance Conference, Open 2012, 2012.

[13] R. Andrews and J. M. Pearce, "The effect of spectral albedo on amorphous silicon and crystalline silicon solar photovoltaic device performance," Under Review, 2012.

[14] R. Andrews, J. M. Pearce, and A. Pollard, "Improved parametric empirical determination of module short circuit current for modelling and optimization of solar photovoltaic systems," Solar Energy, vol. (in press) DOI http://dx.doi.org/10.1016/j.solener.2012.04.016, 2012.

[15] "Kingston climate weather station," Latitude: 4413'24.000" N,Longitude: 7635'58.000" W, 2012. [Online]. Available: http://www.climate.weatheroffice.gc. ca/climateData/dailydata_e.html?StationID $=47267 \&$ Month $=$ $4 \&$ Day $=23 \&$ Year $=2012 \&$ timeframe $=2$

[16] D. L. King, W. E. Boyson, and J. A. Kratochvil, "Photovoltaic array performance model," Sandia, vol. SAND2004-3535, Aug. 2004.

[17] P. J. Brockwell and R. A. Davis, Introduction to time series and forecasting. Springer, 2002.

[18] R. Pfister and M. Schneebeli, "Snow accumulation on boards of different sizes and shapes," Hydrological Processes, vol. 13, no. 14-15, pp. 2345-2355, 1999. [Online]. Available: http://dx.doi.org/10.1002/(SICI)1099-1085(199910) 13:14/15〈2345::AID-HYP873>3.0.CO;2-N 